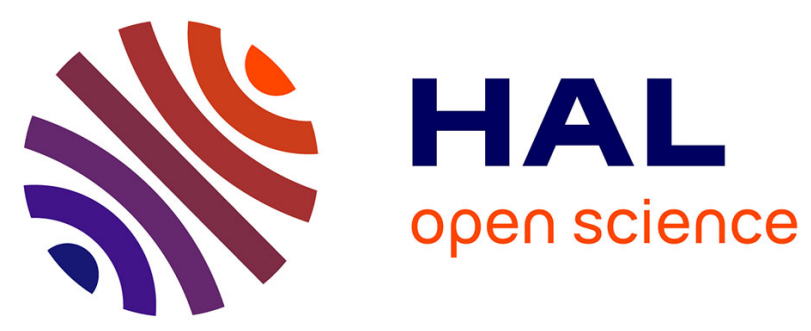

\title{
Structure and Properties of Dimorphic CePdZn
}

\author{
Rainer Poettgen, Wilfried Hermes, Dirk Johrendt, Helen Müller, Ratikanta \\ Mishra
}

\section{To cite this version:}

Rainer Poettgen, Wilfried Hermes, Dirk Johrendt, Helen Müller, Ratikanta Mishra. Structure and Properties of Dimorphic CePdZn. Journal of Inorganic and General Chemistry / Zeitschrift für anorganische und allgemeine Chemie, 2009, 635 (4-5), pp.660. 10.1002/zaac.200900054 . hal-00482073

\section{HAL Id: hal-00482073 https://hal.science/hal-00482073}

Submitted on 8 May 2010

HAL is a multi-disciplinary open access archive for the deposit and dissemination of scientific research documents, whether they are published or not. The documents may come from teaching and research institutions in France or abroad, or from public or private research centers.
L'archive ouverte pluridisciplinaire HAL, est destinée au dépôt et à la diffusion de documents scientifiques de niveau recherche, publiés ou non, émanant des établissements d'enseignement et de recherche français ou étrangers, des laboratoires publics ou privés. 


\section{Structure and Properties of Dimorphic CePdZn}

\begin{tabular}{|r|l|}
\hline Journal: & Zeitschrift für Anorganische und Allgemeine Chemie \\
\hline Manuscript ID: & zaac.200900054 \\
\hline Diley - Manuscript type: & Article \\
\hline Complete List of Authors: & $\begin{array}{l}\text { Poettgen, Rainer; Westf. Wilhelms-Universitaet, Inst. f. Anorgan. u. } \\
\text { Analyt. Chemie } \\
\text { Hermes, Wilfried } \\
\text { Johrendt, Dirk } \\
\text { Müller, Helen } \\
\text { Mishra, Ratikanta }\end{array}$ \\
\hline Keywords: & cerium, dimorphism \\
\hline
\end{tabular}

\section{s scholarONE" \\ Manuscript Central}




\title{
Structure and Properties of Dimorphic CePdZn
}

\author{
Wilfried Hermes $^{\mathrm{a}}$, Ratikanta Mishra ${ }^{\mathrm{a}, \mathrm{b}}$, Helen Müller ${ }^{\mathrm{c}}$, Dirk Johrendt ${ }^{\mathrm{c}}$ *, Rainer \\ Pöttgen $^{\mathrm{a}, *}$ \\ a Münster, Institut für Anorganische und Analytische Chemie der Westfälischen \\ Wilhelms-Universität and NRW Graduate School of Chemistry \\ b Mumbay/India, Bhaba Atomic Research Center \\ c München, Department Chemie und Biochemie der Ludwig-Maximilians-Universität
}

Received,

Dedicated to Professor Reinhard Nesper on the occasion of his $60^{\text {th }}$ birthday

\begin{abstract}
Two modifications of CePdZn have been synthesized and structurally characterized. The high-temperature (HT) $\beta$-CePdZn modification forms upon quenching from the melt. The $\beta$-CePdZn structure adopts the orthorhombic TiNiSi type and transforms to the low-temperature (LT) $\alpha$-CePdZn modification ( $\mathrm{ZrNiAl}$ type) upon annealing at $1080 \mathrm{~K}$. Both modifications have been studied by X-ray powder and single crystal data: $P 6 ;^{-} 2 m, a=740.4(1), c=402.18(5) \mathrm{pm}, \mathrm{wR} 2=0.0427,237 \mathrm{~F}^{2}$ values and 14 variables for $\alpha-C e P d Z n$ and Pnma, $a=707.2(2), b=441.4(2), c=806.3(3) \mathrm{pm}$, wR2 = 0.0827, $469 \mathrm{~F}^{2}$ values and 20 variables for $\beta-\mathrm{CePdZn}$. The palladium and zinc atoms build up three-dimensional $[\mathrm{PdZn}]$ networks in both modifications which leave channels for the cerium atoms. Both modifications show stable trivalent cerium. $\beta$-CePdZn orders antiferromagnetically at 3.2(1) $\mathrm{K}$ while no magnetic ordering has been observed for $\alpha$ $\mathrm{CePdZn}$ down to $2 \mathrm{~K}$. The course of the magnetic data points to stronger $\mathrm{Ce}(4 f)-\operatorname{Pd}(4 d)$ hybridization in $\alpha-C e P d Z n$. Electronic structure calculations reveal only small differ-
\end{abstract}


ences between $\alpha$ - and $\beta$-CePdZn regarding the total energy and the $\mathrm{Ce}(4 \mathrm{f})-\mathrm{Pd}(4 \mathrm{~d})$ hybridization.

Running title: Structure and Properties of Dimorphic CePdZn

Keywords: Cerium, Crystal Chemistry, Phase Transition

\author{
* Prof. Dr. Rainer Pöttgen \\ Institut für Anorganische und Analytische Chemie, Universität Münster \\ Corrensstrasse 30, D-48149 Münster, Germany \\ e-mail: pottgen@uni-muenster.de \\ Dr. Ratikanta Mishra \\ Applied Chemistry Division, Bhabha Atomic Research Centre \\ Trombay, Mumbai-400 085, India \\ e-mail: mishrar@barc.gov.in \\ * Prof. Dr. Dirk Johrendt \\ Department Chemie und Biochemie, Universität München \\ Butenandtstrasse 5-13 (Haus D), D-81377 München, Germany \\ e-mail: dirk.johrendt@cup.uni-muenchen.de
}

\title{
Introduction
}

The equiatomic cerium compounds $\operatorname{Ce} T X(T=$ late transition metal; $X=$ element of the $3^{\text {rd }}, 4^{\text {th }}$, and $5^{\text {th }}$ main group, $\mathrm{Mg}, \mathrm{Zn}, \mathrm{Cd}$ ) have intensively been studied in the last thirty years with respect to their crystal chemistry and greatly varying magnetic and electrical properties, i.e. magnetic ordering with high-ordering temperatures, valence fluctuations, heavy-fermion behaviour, Kondo semimetals or semiconductors, non-Fermi liquid behaviour or quantum phase transitions. An important parameter that governs the electronic and magnetic ground states is the hybridization between the $4 f(\mathrm{Ce})$ orbitals and the conduction electrons.

Most of the CeTX structures are superstructures or intergrowth variants which derive from the aristotypes $\mathrm{AlB}_{2}$ and/or $\mathrm{Fe}_{2} \mathrm{P}$ and their structures are stable over a wide temperature range. Recently, for some CeTX structures, structural transitions driven by temperature, pressure, or the cerium valence have been reported. To give some examples, under hydrostatic pressure of $8.7 \mathrm{GPa}$ the germanide CeAuGe shows a first order phase transition from a hexagonal NdPtSb type to an orthorhombic TiNiSi type structure [1]. 
CePtSn transforms to a ZrNiAl type high-pressure modification under 9.2 GPa and 1325 K [2] and CePdSn [3] shows similar phase transformation. Dimorphism of CePdAl is driven by temperature. The $\mathrm{ZrNiAl}$ type high-temperature modification [4] transforms to a low-temperature (LT) modification with a new structure type upon annealing the sample at $1170 \mathrm{~K}$ [5]. One of the most interesting compounds is the Kondo insulator CeRhAs $[6,7]$ which shows three successive temperature driven phase transition associated with a charge-density-wave and two different modulation vectors. The stannide CeRuSn [8] is a static intermediate-valent system which shows trivalent intermediate-valent ordering at room temperature and a transition to a modulated structure at low temperature. The latter is associated with a temperature-driven valence change of cerium.

In the corresponding equiatomic systems with zinc, so far only X-ray powder data of CeTZn $(T=\mathrm{Cu}, \mathrm{Ag})[9,10], \mathrm{CeNiZn}$ and CePdZn [11, 12], and single crystal data of CePtZn [13], CeAuZn [12], CeZnGe [14, 15], CeZnSn [16, 17], and CeRhZn [18] have been reported. The nickel and palladium compound crystallize with the hexagonal ZrNiAl type structure while CePtZn adopts the TiNiSi type. For CeCuZn $[9,10]$ and CeAgZn [9] the orthorhombic $\mathrm{KHg}_{2}$ type, space group Imma, has been reported with a statistical occupancy of the $8 h$ mercury site. Especially for the copper series it is hard to detect the $\mathrm{Cu}-\mathrm{Zn}$ order by $\mathrm{X}$-ray diffraction since both elements differ by one electron only. CeZnGe and CeZnSn crystallize in the well known YPtAs type structure. CeRhZn [18] adopts the LaNiAl type. In the course of our systematic studies on the magnetic properties of CeTX intermetallics we have re-examined CePdZn. Crystal growth experiments at different temperatures revealed dimorphism with a TiNiSi type hightemperature (HT) modification. Herein we report on the single crystal structure refinements and magnetic properties of both modifications.

\section{Experimental}

\section{Synthesis}

Starting materials for the synthesis of LT- and HT-CePdZn were a cerium ingot (Johnson Matthey), palladium powder (Degussa-Hüls, ca. $200 \mathrm{mesh}$ ), and zinc granules (Merck), all with a stated purity better than $99.9 \%$. Pieces of the cerium ingot were first 
arc-melted [19] into small buttons under purified argon. The argon was purified before with molecular sieves, silica gel and titanium sponge (900 K). The elements were then weighed in the ideal 1:1:1 atomic ratio and arc-welded in small tantalum tubes. The tantalum ampoules were placed in a water-cooled sample chamber of an induction furnace (Hüttinger Elektronik, Freiburg, Typ TIG 1.5/300) [20], rapidly heated to $1370 \mathrm{~K}$ and kept at that temperature for $5 \mathrm{~min}$. The temperature was controlled through a Sensor Therm Metis MS09 pyrometer with an accuracy of $\pm 30 \mathrm{~K}$. The sample was then quenched by switching off the power supply. This annealing sequence was repeated two times, leading to the TiNiSi type high-temperature modification. The silvery brittle sample could easily be separated from the tantalum tube. No reaction with the container material was evident.

For transformation to the low-temperature phase, powder of HT-CePdZn was coldpressed to a small pellet $(\varnothing 6 \mathrm{~mm})$ and placed in a small tantalum crubible in a sealed silica ampoule. The latter was annealed at $1080 \mathrm{~K}$ for two weeks. Alternatively LTCePdZn can be prepared directly from the elements in a tantalum tube in the highfrequency furnace. The sample was first rapidly annealed at $1370 \mathrm{~K}$, followed by an annealing sequence of $6 \mathrm{~h}$ at $970 \mathrm{~K}$. The much shorter reaction time of the induction melting is due to the higher diffusion rates in the oscillating high-frequency field.

\section{X-ray Image Plate Data and Data Collection}

All samples were characterized through Guinier powder patterns using $\mathrm{CuK} \alpha_{1}$ radiation and $\alpha$-quartz ( $a=491.30$ and $c=540.46 \mathrm{pm}$ ) as an internal standard. The Guinier camera was equipped with a Fujifilm / BAS-1800 image plate system. The lattice parameters were refined from the powder data (Table 1). The correct indexing was ensured by comparison of the experimental patterns with calculated ones [21] taking the atomic positions obtained from the structure refinement. The experimental patterns are presented in Figure 1. Our powder lattice parameters for LT-CePdZn are in good agreement with the data originally reported by Iandelli $(a=739.9, c=401.4 \mathrm{pm})$ [11].

Irregularly shaped single crystals were selected from both samples by mechanical fragmentation. They were investigated by Laue photographs on a Buerger camera (white molybdenum radiation; imaging plate technique, Fujifilm, BAS-1800) in order to check 
the quality for intensity data collection. Intensity data of $\alpha-\mathrm{CePdZn}$ were collected at room temperature by use of a four-circle diffractometer (CAD4) with graphite monochromatized MoKa $(71.073 \mathrm{pm})$ radiation and a scintillation counter with pulse height discrimination. Scans were taken in the $\omega / 2 \theta$ mode. An empirical absorption correction was applied on the basis of $\Psi$-scan data, accompanied by a spherical absorption correction. Data of $\beta-C e P d Z n$ were collected at room temperature by use of a Stoe IPDS-II image plate system (graphite monochromatized Mo radiation; $\lambda=71.073 \mathrm{pm}$ ) in oscillation mode and a numerical absorption correction was applied to this data set. All relevant crystallographic data and details of the data collections are listed in Table 1.

The single crystals of $\alpha$ - and $\beta-C e P d Z n$ investigated on the diffractometer and the bulk samples were studied by energy dispersive analyses of X-rays (EDX) using a Leica $420 \mathrm{i}$ scanning electron microscope with $\mathrm{CeO}_{2}$, palladium, and zinc as standards. The experimentally observed compositions were close to the ideal one and no impurity elements were observed.

\section{Physical Property Measurements}

12.42 and $14.61 \mathrm{mg}$ of the $\alpha$ - and $\beta-\mathrm{CePdZn}$ samples were packed in kapton foil and attached to the sample holder rod of a VSM for measuring the magnetic properties in a Quantum Design Physical-Property-Measurement-System in the temperature range 2.1$305 \mathrm{~K}$ with magnetic flux densities up to $80 \mathrm{kOe}$. For heat capacity $\left(\mathrm{C}_{\mathrm{p}}\right)$ measurements $(2.1-50 \mathrm{~K})$ the samples were glued to the platform of a pre-calibrated heat capacity puck using Apiezon $N$ grease.

\section{Computational Details}

The total energies and atomic structure calculations have been performed with the Vienna ab initio Simulation Package (VASP) [22], based on density functional theory. The all electron PAW method was used [23] and the cut-off energy for the plane-wave expansion was $375 \mathrm{eV}$. Convergence limits were $1 \times 10^{-6} \mathrm{eV}$ per formula unit (total energy) and $5 \times 10^{-4} \mathrm{eV}^{-1}$ (forces). Exchange and correlation were treated by the local density approximation (LDA) proposed by Perdew and Zunger [24]. In addition the generalized-gradient approximation (GGA) of Perdew, Burke and Ernzerhof [25] was used. Integration in the Brillouin zone were done by the tetrahedron method including Blöchl 
correction [26] using a $6 \times 6 \times 10$ mesh of $42 k$ points for $\alpha$-CePdZn and a $5 \times 8 \times 6$ mesh of $60 k$ points for $\beta-C e P d Z n$.

\section{Results and Discussion}

\section{Structure Refinements}

The isotypy of $\alpha-\mathrm{CePdZn}$ with the hexagonal $\mathrm{ZrNiAl}$ type [27] and $\beta-\mathrm{CePdZn}$ with the orthorhombic TiNiSi type [28] was already evident from the Guinier powder data. Consequently, the diffractometer data sets were in agreement with space groups $P 6 ;{ }^{-} 2 m$ $(\alpha-\mathrm{CePdZn})$ and Pnma $(\beta-\mathrm{CePdZn})$. The atomic positions of NP- and HP-CePdSn [3] were taken as starting atomic parameters and both structures were refined using SHELXL-97 [29] (full-matrix least-squares on $\mathrm{F}^{2}$ ) with anisotropic atomic displacement parameters for all atoms. The occupancy parameters were refined in separate series of least-squares cycles in order to check for deviations from the ideal composition. All sites were fully occupied within two standard uncertainties and in the final cycles the ideal occupancy parameters were assumed again. Refinement of the correct absolute structure for $\alpha-C e P d Z n$ was ensured through calculation of the Flack parameter [30, 31]. Final difference Fourier synthesis revealed no significant residual peaks. The positional parameters and interatomic distances are listed in Tables 2 and 3. Further details on the structure refinements are available.*

\footnotetext{
* Details may be obtained from: Fachinformationszentrum Karlsruhe, D-76344 Eggenstein-Leopoldshafen (Germany), by quoting the Registry No's. CSD-420208 ( $\alpha$ CePdZn) and CSD-420207 ( $\beta$-CePdZn).
}

\section{Crystal Chemistry}

The structures of the low- and high-temperature modification of CePdZn have been refined from single crystal diffractometer data. So far, only X-ray powder data have been reported for the hexagonal modification by Iandelli [11]. The corresponding structure types $\mathrm{ZrNiAl}$ [27] for $\alpha-\mathrm{CePdZn}$ and TiNiSi [28] for $\beta$-CePdZn have repeatedly been discussed in literature [32-38]. For further details we refer to these overviews and 
the recent work on dimorphic CePtSn [2] and $\mathrm{YbPdSn}[39,40]$. Herein we focus only on the peculiarities for dimorphic CePdZn.

The cerium near neighbour coordination of $\alpha$ - and $\beta-\mathrm{CePdZn}$ is presented in Figure 2. Both cerium sites have five nearest palladium neighbors. This coordination would have been expected from the course of the electronegativities. In $\alpha-\mathrm{CePdZn}$ the average Ce-Pd distance $(301.5 \mathrm{pm})$ is somewhat smaller than in $\beta$-CePdZn $(307.5 \mathrm{pm})$ and one can expect stronger $\mathrm{Ce}(4 \mathrm{f})-\mathrm{Pd}(4 \mathrm{~d})$ hybridization in $\alpha-\mathrm{CePdZn}$, as supported by the magnetic and specific heat data (vide infra). The Ce-Pd distances are all slightly longer than the sum of the covalent radii [41] of 293 pm. The Ce-Ce distances in both modifications are well above the Hill limit [42] for f electron localization of $340 \mathrm{pm}$.

\section{Quantum chemical calculations}

The crystal structures of $\alpha-\mathrm{CePdZn}$ and $\beta-\mathrm{CePdZn}$ were optimized by using LDA and GGA functionals. The optimized lattice parameters are presented in Table 4. The volume per formula unit calculated for $\alpha-\mathrm{CePdZn}$ is slightly smaller than for $\beta-\mathrm{CePdZn}$ in agreement with the experimental data, thus indeed the low temperature modification shows a slightly smaller unit cell volume as the high temperature modification. The calculated total energies (Table 5) of the high and low temperature modifications are almost the same $( \pm 1 \mathrm{~kJ} / \mathrm{mol})$ within the LDA $(\beta-C e P d Z n$ more stable) and GGA $(\alpha-$ CePdZn more stable), and thus allow no identification of one polymorph to be the most stable. The calculated densities of states (DOS) of $\alpha$ - and $\beta$-CePdZn (Figure 6) clearly reveal hybridization of $\mathrm{Ce}(4-f)$ and $\mathrm{Pd}(4-d)$ states for both modifications. However, the difference of this hybridization is rather small and allows no reliable quantification.

\section{Magnetic susceptibility and specific heat}

In the literature only the magnetic properties of $\mathrm{CePd}_{2} \mathrm{Zn}_{3}$ in the ternary intermetallic $\mathrm{Ce}-\mathrm{Pd}-\mathrm{Zn}$ system have been described so far. $\mathrm{CePd}_{2} \mathrm{Zn}_{3}$ shows paramagnetic behaviour with stable trivalent cerium and no hint for magnetic ordering was observed down to 2.1 K [43].

The temperature dependence of the magnetic $(\chi=\mathrm{M} / \mathrm{H})$ and the inverse magnetic susceptibility $\chi^{-1}(\mathrm{~T})$ of $\alpha-\mathrm{CePdZn}$ and $\beta-\mathrm{CePdZn}$ are displayed in Figure 3, measured 
while warming in a $d c$ field of $10 \mathrm{kOe}$ after zero field cooling each sample to the lowest available temperature. For both compounds studied here, $\chi$ increases with decreasing temperature. Down to $3.1 \mathrm{~K}$, the $H=10 \mathrm{kOe}$ susceptibility data give no hint for magnetic ordering in both modifications. Both compounds show Curie-Weiss behavior in the temperature range $75-300 \mathrm{~K}$. A fit of the susceptibility data in this temperature range revealed an effective magnetic moment of $\mu_{\mathrm{eff}}=2.42(1) \mu_{\mathrm{B}} / \mathrm{Ce}$ atom and a paramagnetic Curie temperature of $\theta_{\mathrm{P}}=-39.6(1) \mathrm{K}$ for $\alpha-\mathrm{CePdZn}$ and $\mu_{\text {eff }}=2.54(1) \mu_{\mathrm{B}} / \mathrm{Ce}$ atom and $\theta_{\mathrm{P}}=3.3(1) \mathrm{K}$ for $\beta-\mathrm{CePdZn}$. These $\mu_{\text {eff }}$ values are close to the free ion value of $2.54 \mu_{\mathrm{B}}$ for $\mathrm{Ce}^{3+}$, thus indicating purely trivalent cerium in both modifications. The $\theta_{\mathrm{P}}$ values indicate that the magnetic interaction is of a ferromagnetic type in $\beta$-CePdZn and of an antiferromagnetic type in $\alpha-C e P d Z n$. $\beta$-CePdZn follows almost a Curie law. The deviation of $1 / \chi$ vs $\mathrm{T}$ in $\alpha-\mathrm{CePdZn}$ at low temperatures may be influenced by crystal electric field splitting of the $J=5 / 2$ ground state of $\mathrm{Ce}^{3+}$ and a thermally induced change of the $4 f$ electron level occupancy and/or the beginning of short-range magnetic fluctuations, as frequently observed in related cerium intermetallics, e.g. $\mathrm{Ce} T \mathrm{~Pb}(T=\mathrm{Cu}, \mathrm{Pd}$, $\mathrm{Ag}, \mathrm{Au}$ ) [44] and CePtZn [13]. It is important to note that the susceptibility $\chi_{\mathrm{m}}$ (measured at $10 \mathrm{kOe}$ ) of $\beta-\mathrm{CePdZn}$ at $3 \mathrm{~K}$ is almost four times larger than that determined for $\alpha-\mathrm{CePdZn}$. In other words, the magnetic correlations in $\beta-\mathrm{CePdZn}$ are enhanced. An anomaly at around 3.4(1) $\mathrm{K}$ is clearly visible for the high temperature modification in the $\chi(\mathrm{T})$ curve measured at a low field of 100 Oe (inset of Figure 3). Both the ZFC and the FC data show an antiferromagnetic transition at $\mathrm{T}_{\mathrm{N}}=3.4(1) \mathrm{K}$, they do not bifurcate and are the same within the experimental errors. For $\alpha-C e P d Z n$ we could not observed a magnetic transition down to $2.1 \mathrm{~K}$ measured at a low field of $100 \mathrm{Oe}$.

Due to the abased susceptibility in $\alpha-C e P d Z n$, the smaller value of $\mu_{\text {eff }}$ in $\alpha-C e P d Z n$, the observed antiferromagnetic ordering at 3.4(1) $\mathrm{K}$ in $\beta-\mathrm{CePdZn}$ and no ordering in $\alpha$ CePdZn, we can establish an increase of the $J_{\text {cf }}$ interaction from the high temperature modification to the low temperature modification, enabling stronger Ce $(4 f)-\mathrm{Pd}(4 d)$ hybridization. This is in line with the crystal chemical data (vide ultra).

In Figure 4 we present the magnetization data for $\alpha-C e P d Z n$ and $\beta-C e P d Z n$ measured in $\mathrm{ZFC}$ state at 5,10 and $15 \mathrm{~K}$. The magnetizations for $\alpha-\mathrm{CePdZn}$ and $\beta-\mathrm{CePdZn}$ vary 
almost in a linear fashion at 10 and $15 \mathrm{~K}$ as expected for a paramagnetic material. At 5 $\mathrm{K}$ the curvature becomes more pronounced for both samples, but stronger for the hightemperature modification. $\beta-\mathrm{CePdZn}$ shows a tendency for saturation at high fields. $M$ $(\mathrm{T}=5 \mathrm{~K})$ varies linearly with initial application of the field and reveals a curvature above $20 \mathrm{kOe}$, clearly indicating the antiferromagnetic nature of magnetic ordering in $\beta$ CePdZn. It may be noted here that, for both modifications, the maxium moment observed at $80 \mathrm{kOe}$ and $5 \mathrm{~K}$ does not reach the expected moment value of $2.14 \mu_{\mathrm{B}} / \mathrm{Ce}$ atom (according to $g \times J$ ). The values for $\alpha-\mathrm{CePdZn}$ and $\beta-\mathrm{CePdZn}$ are 0.4 and 1.2 $\mu_{\mathrm{B}} / \mathrm{Ce}$ atom, respectively. The decrease in the saturation moment can be attributed to crystal field effects (see also the deviation of $\chi^{-1}(\mathrm{~T})$ from the Curie-Weiss fit, espessially in $\alpha-\mathrm{CePdZn}$ ), and has been observed for many other cerium intermetallics [45, 46, and refs. therein]. The smaller value of the observed moment in $\alpha-C e P d Z n$ is in line with a stronger $\mathrm{Ce}(4 f)-\mathrm{Pd}(4 d)$ hybridization, in agreement with the structural data (vide ul$\operatorname{tra})$.

The specific heat $\left(\mathrm{C}_{\mathrm{P}}\right)$ data are plotted in Fig. 5. The magnetic ordering temperature (Néel temperature) of $\beta$-CePdZn is characterized by a $\lambda$-like anomaly. The ordering temperature is 3.2(1) $\mathrm{K}$, in good agreement with the magnetic data. $\alpha-\mathrm{CePdZn}$ does not order magnetically above $2.1 \mathrm{~K}$.

We thank Dipl.-Ing. U. Ch. Rodewald for the intensity data collections. This work was financially supported by the Deutsche Forschungsgemeinschaft. R.M. is indebted to the Alexandervon-Humboldt Foundation for a research stipend. The PhD thesis of W.H. is supported by the Fonds der Chemischen Industrie.

\section{References}

[1] V. Brouskov, M. Hanfland, R. Pöttgen, U. Schwarz, Z. Kristallogr. 2005, 220, 122.

[2] J. F. Riecken, G. Heymann, T. Soltner, R.-D. Hoffmann, H. Huppertz, D. Johrendt, R. Pöttgen, Z. Naturforsch. 2005, 60b, 821.

[3] G. Heymann, J. F. Riecken, S. Rayaprol, S. Christian, R. Pöttgen, H. Huppertz, Z. Anorg. Allg. Chem. 2007, 633, 77.

[4] F. Hulliger, J. Alloys Compd. 1995, 218, 44. 
[5] A. Gribanov, A. Tursina, E. Murashova, Y. Seropegin, E. Bauer, H. Kaldarar, R. Lackner, H. Michor, E. Royanian, M. Reissner, P. Rogl, J. Phys.: Condens. Matter 2006, 18, 9593.

[6] T. Sasakawa, T. Suemitsu, T. Takabatake, Y. Bando, K. Uemo, M. H. Jung, M. Sera, T. Suzuki, T. Fujita, M. Nakajima, K. Iwasa, M. Kohgi, Ch. Paul, St. Berger, E. Bauer, Phys. Rev. B 2002, 66, 041103.

[7] K. Uemo, K. Masumori, T. Sasakawa, F. Iga, T. Takabatake, Y. Ohishi, T. Adachi, Phys. Rev. B 2005, 71, 064110.

[8] J. F. Riecken, W. Hermes, B. Chevalier, R.-D. Hoffmann, F. M. Schappacher, R. Pöttgen, Z. Anorg. Allg. Chem. 2007, 633, 1094.

[9] M. L. Fornasini, A. Iandelli, F. Merlo, M. Pani, Intermetallics, 2000, 8, 239.

[10] P. Morin, D. Gignoux, J. Voiron, A. P. Murani, Physica B, 1992, 180\&181, 173.

[11] A. Iandelli, J. Alloys Compd. 1992, 182, 87.

[12] W. Hermes, R. Mishra, U. Ch. Rodewald, R Pöttgen, Z. Naturforsch., 2008, 63b, 537.

[13] R. Mishra, W. Hermes, R. Pöttgen, Z. Naturforsch. 2007, 62b, 1581.

[14] M. Pani, P. Manfrinetti, A. Palenzona, Intermetallics, 2009, 17, \#\#\#. Doi:10.1016/j.intermet.2008.10.008

[15] W. Hermes, R. Pöttgen, Z. Naturforsch. 2009, 64b, in press.

[16] P. Manfrinetti, M. Pani, J. Alloys Compd. 2005, 393, 180.

[17] W. Hermes, S. F. Matar, T. Harmening, U. Ch. Rodewald, M. Eul, R. Pöttgen, Z. Naturforsch. 2009, 64b, in press.

[18] W. Hermes, A. F. Al Alam, S. F. Matar, R. Pöttgen, Solid State Sci., 2008, 10, 1895.

[19] R. Pöttgen, Th. Gulden, A. Simon, GIT Labor Fachzeitschrift 1999, 43, 133.

[20] D. Kußmann, R.-D. Hoffmann, R. Pöttgen, Z. Anorg. Allg. Chem. 1998, 624, 1727.

[21] K. Yvon, W. Jeitschko, E. Parthé, J. Appl. Crystallogr. 1977, 10, 73.

[22] G. Kresse, J. Furthmüller, Comput. Mater. Sci. 1996, 6, 15.

[23] G. Kresse, D. Joubert, Phys. Rev. B 1999, 59, 1758.

[24] J. P. Perdew, A. Zunger, Phys. Rev. B 1981, 23, 5048.

[25] J. P. Perdew, S. Burke, M. Ernzerhof, Phys. Rev. Lett. 1996, 77, 3865.

[26] P. E. Blöchl, Phys. Rev. B 1994, 50, 17953.

[27] M. F. Zumdick, R.-D. Hoffmann, R. Pöttgen, Z. Naturforsch. 1999, 54b, 45.

[28] C. B. Shoemaker, D. P. Shoemaker, Acta Crystallogr. 1965, 18, 900. 
[29] G. M. Sheldrick, SHELXL-97 - Program for Crystal Structure Refinement, University of Göttingen, Germany 1997.

[30] H. D. Flack, G. Bernadinelli, Acta Crystallogr. A 1999, 55, 908.

[31] H. D. Flack, G. Bernadinelli, J. Appl. Crystallogr. 2000, 33, 1143.

[32] G. Nuspl, K. Polborn, J. Evers, G. A. Landrum, R. Hoffmann, Inorg. Chem. 1996, 35,6922 .

[33] G. A. Landrum, R. Hoffmann, J. Evers, H. Boysen, Inorg. Chem. 1998, 37, 5754.

[34] R.-D. Hoffmann, R. Pöttgen, Z. Kristallogr. 2001, 216, 127.

[35] M. D. Bojin, R. Hoffmann, Helv. Chim. Acta 2003, 86, 1653.

[36] M. D. Bojin, R. Hoffmann, Helv. Chim. Acta 2003, 86, 1683.

[37] E. Parthé, L. Gelato, B. Chabot, M. Penzo, K. Cenzual, R. Gladyshevskii, TYPIXStandardized Data and Crystal Chemical Characterization of Inorganic Structure Types. Gmelin Handbook of Inorganic and Organometallic Chemistry, 8th edition, Springer, Berlin (Germany) 1993.

[38] M. F. Zumdick, R. Pöttgen, Z. Kristallogr. 1999, 214, 90.

[39] D. Kußmann, R. Pöttgen, B. Künnen, R. Müllmann, B. D. Mosel, G. Kotzyba, Z. Kristallogr. 1998, 613, 356.

[40] T. Görlach, S. Putselyk, A. Hamann, T. Tomanić, M. Uhlarz, F. M. Schappacher, R. Pöttgen, H. v. Löhneysen, Phys. Rev. B 2007, 76, 205112.

[41] J. Emsley, The Elements, Oxford University Press, Oxford (U.K.) 1999.

[42] H. H. Hill, in Plutonium and other Actinides, Mines, W. N. (ed.) Nuclear Materials Series, AIME 1970, 17, 2.

[43] W. Hermes, St. Linsinger, R. Mishra, R. Pöttgen, Monatsh. Chem. 2008, 139, 1143.

[44] W. Hermes, S. Rayaprol, R. Pöttgen, Z. Naturforsch. 2007, 62b, 901.

[45] R. Kraft, R. Pöttgen, D. Kaczorowski, Chem. Mater. 2003, 15, 2998.

[46] R. Movshovich, J. M. Lawrence, M. F. Hundley, J. Neumeier, J. D. Thompson, A. Lacerda, Z. Fisk, Phys. Rev. B 1996, 53, 5456. 
Table 1. Crystal data and structure refinement for $\alpha-$ and $\beta-\mathrm{CePdZn}$.

\begin{tabular}{lll}
\hline Empirical formula & $\alpha$-CePdZn & $\beta$-CePdZn \\
Molar mass & $311.89 \mathrm{~g} / \mathrm{mol}$ & high-temperature phase \\
Space group; $Z$ & $P 6 ; 2 m ; 3$ & $311.89 \mathrm{~g} / \mathrm{mol}$ \\
Structure type & $\mathrm{ZrNiAl}$ & $P n m a ; 4$ \\
Pearson symbol & $\mathrm{hP} 9$ & $\mathrm{TiNiSi}$ \\
Unit cell dimensions & $a=740.4(1) \mathrm{pm}$ & $\mathrm{oP} 12$ \\
(Guinier powder data) & $b=a$ & $a=707.2(2) \mathrm{pm}$ \\
& $c=402.18(5) \mathrm{pm}$ & $b=441.4(2) \mathrm{pm}$ \\
& $V=0.1909 \mathrm{~nm}^{3}$ & $c=806.3(3) \mathrm{pm}$ \\
Calculated density & $8.14 \mathrm{~g} / \mathrm{cm}^{3}$ & $V=0.2517 \mathrm{~nm}^{3}$ \\
Crystal size & $30 \times 40 \times 50 \mu \mathrm{m}^{3}$ & $8.23 \mathrm{~g} / \mathrm{cm}^{3}$ \\
Transm. ratio (max/min) & 1.20 & $20 \times 30 \times 60 \mu \mathrm{m}^{3}$ \\
Absorption coefficient & $33.5 \mathrm{~mm}^{-1}$ & 5.76 \\
F(000) & 402 & $33.9 \mathrm{~mm} \mathrm{~m}^{-1}$ \\
$\theta$ Range & 3 to $30^{\circ}$ & 536 \\
Range in hkl & $\pm 10 ; \pm 10 ; 0-5$ & 3 to $32^{\circ}$ \\
Total no. reflections & 1259 & $\pm 10 ; \pm 6 ; \pm 11$ \\
Independent reflections & $237\left(\mathrm{R}_{\text {int }}=0.1081\right)$ & 5816 \\
Reflections with $I>2 \sigma(I)$ & $205\left(\mathrm{R}_{\text {sigma }}=0.0552\right)$ & $469\left(\mathrm{R}_{\text {int }}=0.1259\right)$ \\
Data / parameters & $237 / 14$ & $400\left(\mathrm{R}_{\text {sigma }}=0.0448\right)$ \\
Goodness-of-fit on $F^{2}$ & 1.092 & $469 / 20$ \\
Final $R$ indices $[I>2 \sigma(I)]$ & $\mathrm{R} 1=0.0315$ & 1.063 \\
& $\mathrm{wR} 2=0.0400$ & $\mathrm{R} 1=0.0332$ \\
$R$ indices (all data) & $\mathrm{R} 1=0.0442$ & $\mathrm{wR} 2=0.0816$ \\
& $\mathrm{wR} 2=0.0427$ & $\mathrm{R} 1=0.0382$ \\
Extinction coefficient & $0.0039(5)$ & $\mathrm{wR} 2=0.0827$ \\
Flack parameter & $0.11(7)$ & $0.009(1)$ \\
Largest diff. peak and hole & $1.62 /-1.58 \mathrm{e} / \AA^{3}$ & - \\
\hline & & $2.59 /-3.84 \mathrm{e} / \AA^{3}$ \\
\hline
\end{tabular}


Table 2. Atomic coordinates and anisotropic displacement parameters $\left(\mathrm{pm}^{2}\right)$ for $\alpha$ - and $\beta$-CePdZn. $U_{\text {eq }}$ is defined as one third of the trace of the orthogonalized $\mathrm{U}_{\mathrm{ij}}$ tensor. The anisotropic displacement factor exponent takes the form $-2 \pi^{2}\left[\left(h a^{*}\right)^{2} U_{11}+\ldots+2 k h a^{*} b^{*} U_{12}\right] . U_{23}=0$.

\begin{tabular}{lcccccccccc}
\hline Atom & $\begin{array}{c}\text { Wyckoff } \\
\text { position }\end{array}$ & $x$ & $y$ & $z$ & $\mathrm{U}_{11}$ & $\mathrm{U}_{22}$ & $\mathrm{U}_{33}$ & $\mathrm{U}_{12}$ & $\mathrm{U}_{13}$ & $\mathrm{U}_{\mathrm{eq}}$ \\
\hline $\boldsymbol{\alpha}$-CePdZn (low-temperature phase) & & & & & & & & \\
$\mathrm{Ce}$ & $3 f$ & $0.4113(2)$ & 0 & 0 & $108(4)$ & $97(6)$ & $97(5)$ & $48(3)$ & 0 & $102(3)$ \\
$\mathrm{Pd} 1$ & $1 a$ & 0 & 0 & 0 & $151(8)$ & $\mathrm{U}_{11}$ & $128(15)$ & $76(4)$ & 0 & $144(6)$ \\
$\mathrm{Pd} 2$ & $2 d$ & $2 / 3$ & $1 / 3$ & $1 / 2$ & $133(6)$ & $\mathrm{U}_{11}$ & $106(11)$ & $67(3)$ & 0 & $124(4)$ \\
$\mathrm{Zn}$ & $3 g$ & $0.7616(3)$ & 0 & $1 / 2$ & $133(11)$ & $125(12)$ & $138(13)$ & $62(6)$ & 0 & $133(6)$ \\
$\boldsymbol{\beta}-\mathbf{C e P d Z n}($ high-temperature phase) & & & & & & & \\
$\mathrm{Ce}$ & $4 c$ & $0.03202(7)$ & $1 / 4$ & $0.69327(7)$ & $150(3)$ & $138(3)$ & $145(3)$ & 0 & $-8(2)$ & $144(2)$ \\
$\mathrm{Pd}$ & $4 c$ & $0.26896(10)$ & $1 / 4$ & $0.39212(10)$ & $166(3)$ & $130(3)$ & $164(4)$ & 0 & $13(2)$ & $153(2)$ \\
$\mathrm{Zn}$ & $4 c$ & $0.65160(16)$ & $1 / 4$ & $0.43125(16)$ & $199(5)$ & $133(5)$ & $137(6)$ & 0 & $-12(4)$ & $156(3)$ \\
\hline
\end{tabular}

Table 3. Interatomic distances (pm), calculated with the powder lattice parameters of $\alpha$ - and $\beta$-CePdZn. Standard deviations are all equal or less than $0.2 \mathrm{pm}$. All distances of the first coordination sphere are listed.

\begin{tabular}{|c|c|c|c|c|c|c|c|}
\hline \multicolumn{4}{|c|}{$\alpha-C e P d Z n$} & \multicolumn{4}{|c|}{$\beta$-CePdZn } \\
\hline \multirow[t]{10}{*}{ Ce: } & 4 & $\mathrm{Pd} 2$ & 300.7 & Ce: & 1 & $\mathrm{Pd}$ & 295.0 \\
\hline & 1 & $\operatorname{Pd} 1$ & 304.5 & & 2 & $\mathrm{Pd}$ & 307.0 \\
\hline & 2 & $\mathrm{Zn}$ & 328.2 & & 2 & $\mathrm{Pd}$ & 314.3 \\
\hline & 4 & $\mathrm{Zn}$ & 332.5 & & 1 & $\mathrm{Zn}$ & 314.3 \\
\hline & 4 & $\mathrm{Ce}$ & 387.3 & & 2 & $\mathrm{Zn}$ & 320.0 \\
\hline & 2 & $\mathrm{Ce}$ & 402.2 & & 2 & $\mathrm{Zn}$ & 329.9 \\
\hline & & & & & 1 & $\mathrm{Zn}$ & 342.1 \\
\hline & & & & & 2 & $\mathrm{Ce}$ & 365.3 \\
\hline & & & & & 1 & $\mathrm{Pd}$ & 382.6 \\
\hline & & & & & 2 & $\mathrm{Ce}$ & 384.6 \\
\hline \multirow[t]{2}{*}{ Pd1: } & 6 & $\mathrm{Zn}$ & 267.6 & Pd: & 2 & $\mathrm{Zn}$ & 268.6 \\
\hline & 3 & $\mathrm{Ce}$ & 304.5 & & 1 & $\mathrm{Zn}$ & 272.4 \\
\hline \multirow[t]{5}{*}{$\operatorname{Pd} 2:$} & 3 & $\mathrm{Zn}$ & 288.4 & & 1 & $\mathrm{Zn}$ & 273.6 \\
\hline & 6 & $\mathrm{Ce}$ & 300.7 & & 1 & $\mathrm{Ce}$ & 295.0 \\
\hline & & & & & 2 & $\mathrm{Ce}$ & 307.0 \\
\hline & & & & & 2 & $\mathrm{Ce}$ & 314.3 \\
\hline & & & & & 1 & $\mathrm{Ce}$ & 382.6 \\
\hline \multirow[t]{8}{*}{$\mathrm{Zn:}$} & 2 & $\operatorname{Pd} 1$ & 267.6 & $\mathrm{Zn}:$ & 2 & $\mathrm{Pd}$ & 268.6 \\
\hline & 2 & $\mathrm{Pd} 2$ & 288.4 & & 1 & $\mathrm{Pd}$ & 272.4 \\
\hline & 2 & $\mathrm{Zn}$ & 305.8 & & 1 & $\mathrm{Pd}$ & 273.6 \\
\hline & 2 & $\mathrm{Ce}$ & 328.2 & & 1 & $\mathrm{Ce}$ & 314.3 \\
\hline & 4 & $\mathrm{Ce}$ & 332.5 & & 2 & $\mathrm{Ce}$ & 320.0 \\
\hline & & & & & 2 & $\mathrm{Zn}$ & 327.1 \\
\hline & & & & & 2 & $\mathrm{Ce}$ & 329.9 \\
\hline & & & & & 1 & $\mathrm{Ce}$ & 342.1 \\
\hline
\end{tabular}

Table 4. Experimental and theoretical lattice parameters of $\alpha$ - and $\beta-\mathrm{CePdZn}$.

\section{$\alpha-C e P d Z n$}

Experiment LDA

\section{$\beta-C e P d Z n$}

GGA Experiment LDA 


\begin{tabular}{lllllll}
\hline volume $\left(\mathrm{nm}^{3}\right)$ & 0.1909 & 0.1726 & 0.1895 & 0.2517 & 0.2278 & 0.2503 \\
$a(\mathrm{pm})$ & $740.4(1)$ & 701.95 & 735.42 & $707.2(2)$ & 666.86 & 699.06 \\
$b(\mathrm{pm})$ & $b=a$ & $b=a$ & $b=a$ & $441.4(2)$ & 437.19 & 442.96 \\
$c(\mathrm{pm})$ & $402.18(5)$ & 404.59 & 404.61 & $806.3(3)$ & 781.37 & 808.45 \\
\hline
\end{tabular}

Table 5. Total energies per formula unit of $\alpha-$ and $\beta-C e P d Z n$.

\begin{tabular}{lcc}
\hline & LDA & GGA \\
$\boldsymbol{\alpha}-C e P d Z n(\mathrm{~kJ} / \mathrm{mol})$ & -1639.03 & -1393.50 \\
$\boldsymbol{\beta}-C e P d Z n(\mathrm{~kJ} / \mathrm{mol})$ & -1640.02 & -1392.33 \\
$\Delta E(\mathrm{~kJ} / \mathrm{mol})$ & 0.99 & -1.17 \\
\hline
\end{tabular}




\section{Figure Captions}

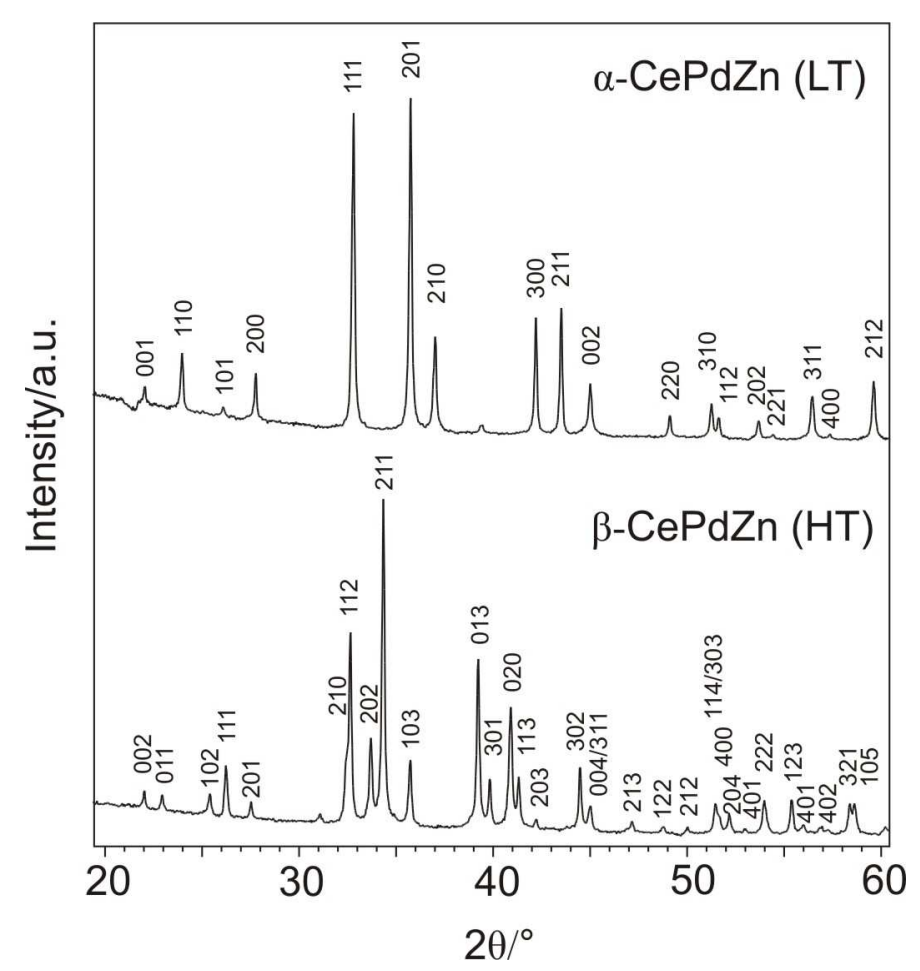

Fig. 1 Guinier powder patterns $\left(\mathrm{CuK \alpha} \alpha_{1}\right.$ radiation) of $\alpha$-CePdZn (LT) and $\beta$ CePdZn (HT). The $h k l$ indices are indicated.
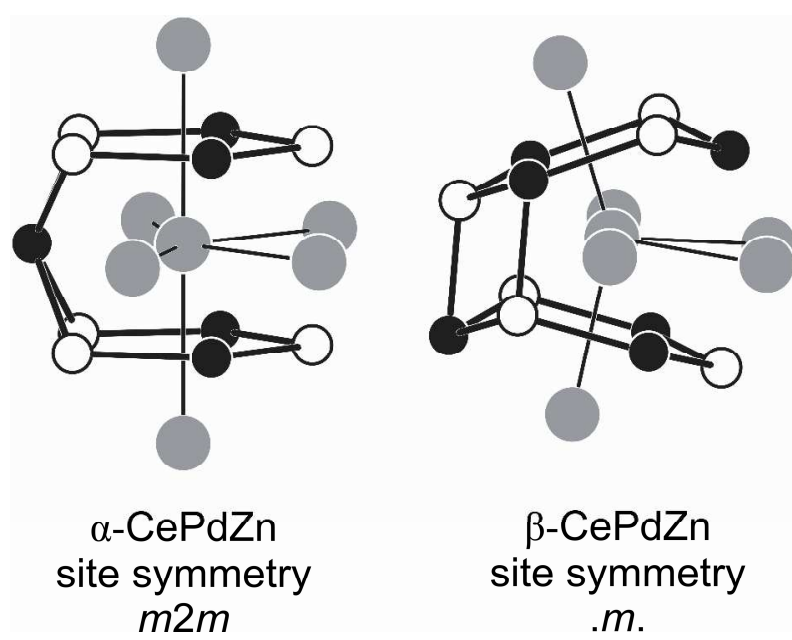

Fig. 2 Coordination polyhedra of the cerium atoms in $\alpha$ - and $\beta$-CePdZn. The cerium, palladium, and zinc atoms are drawn as medium grey, filled, and open circles, respectively. 


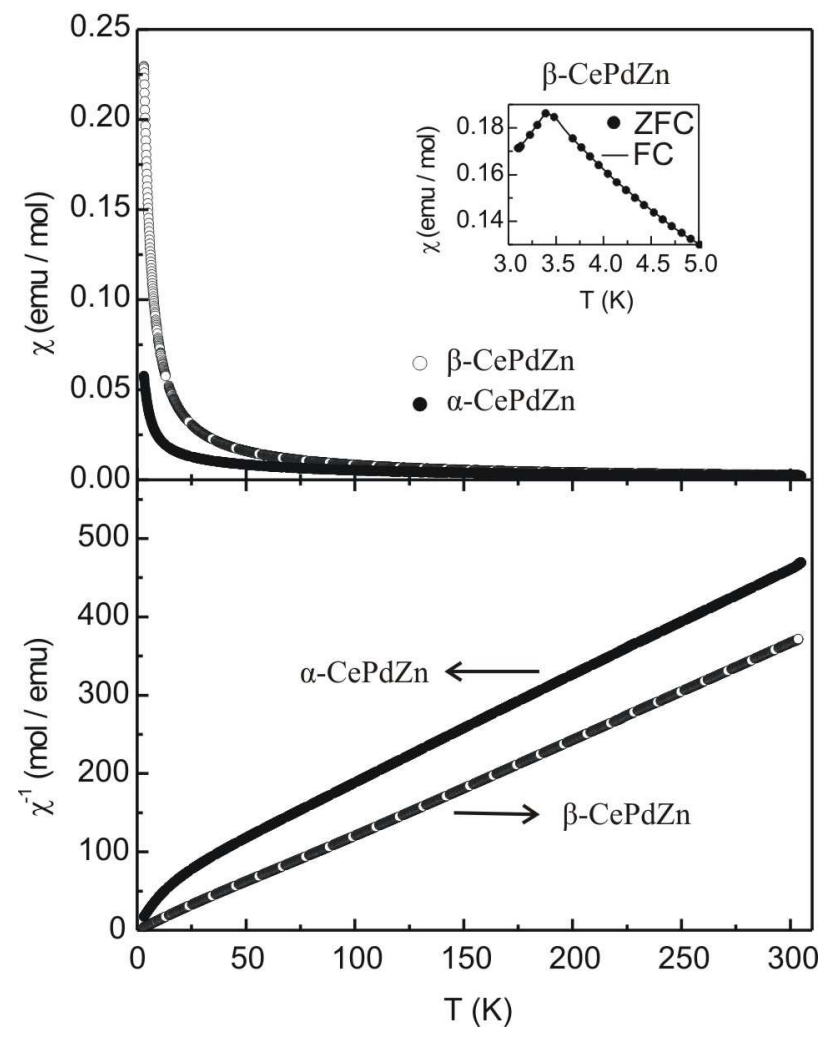

Fig. 3 Temperature dependence of the magnetic $(\chi)$ (top panel) and the inverse magnetic susceptibility $\left(\chi^{-1}\right)$ (bottom panel) of $\alpha-C e P d Z n$ and $\beta-C e P d Z n$ measured at an external flux density of $10 \mathrm{kOe}$. The inset shows $\chi(\mathrm{T})$ for $\beta$-CePdZn measured at $H=100$ Oe after zero field cooling (ZFC) and field cooling (FC) the sample. 


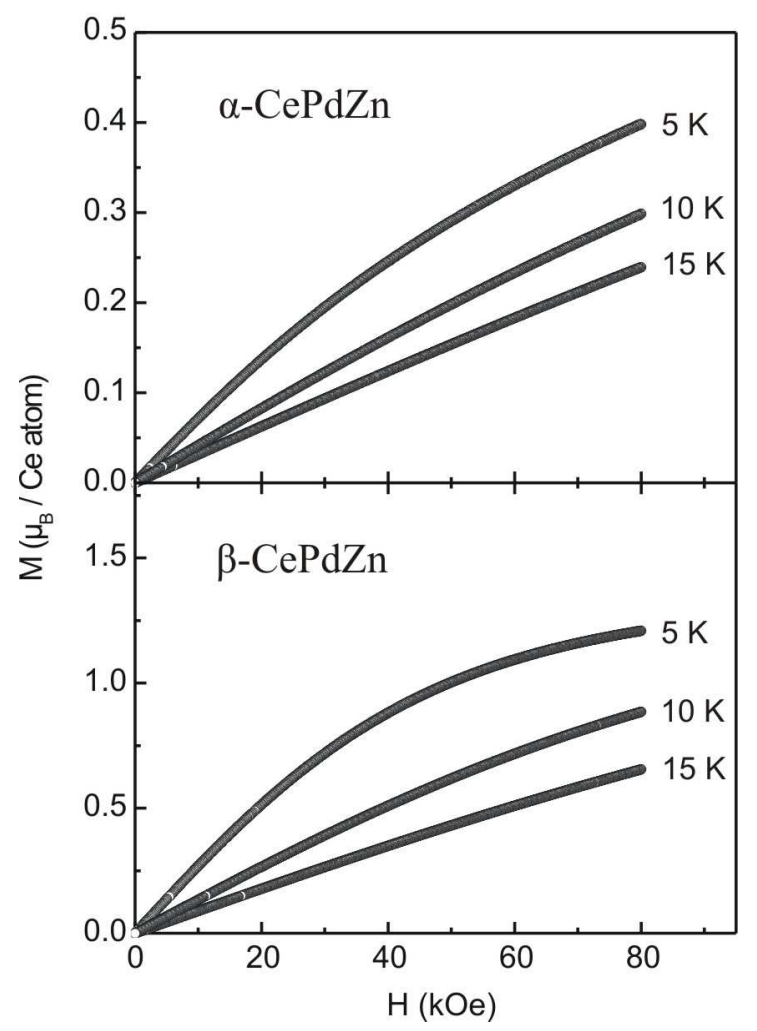

Fig. 4 Magnetization $v s$ external magnetic flux density of $\alpha-C e P d Z n$ and $\beta$ CePdZn at 5, 10, and $15 \mathrm{~K}$. 


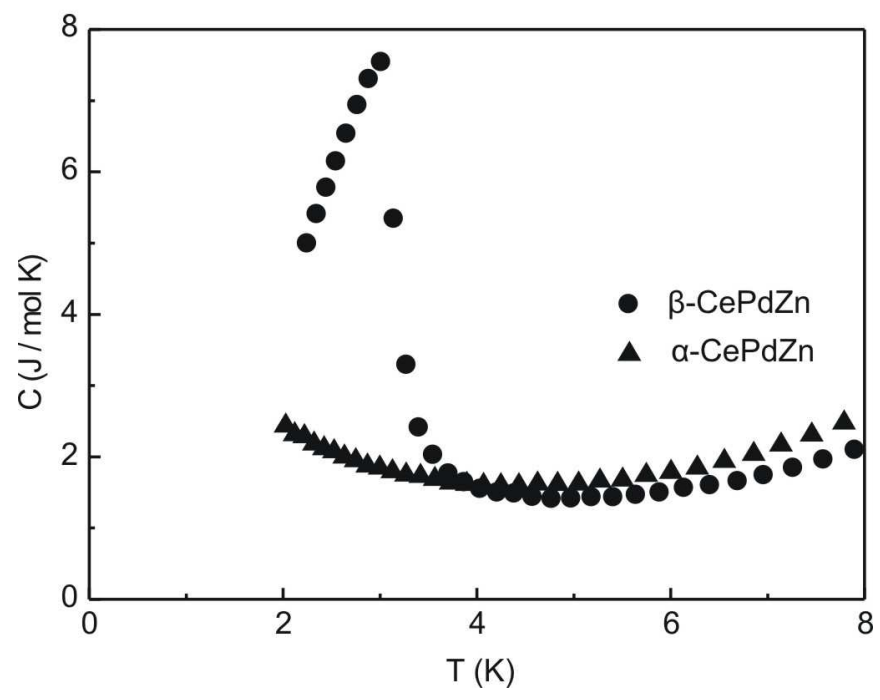

Fig. 5 Temperature dependence of the specific heat of $\alpha-C e P d Z n$ and $\beta-C e P d Z n$ measured in zero magnetic field.
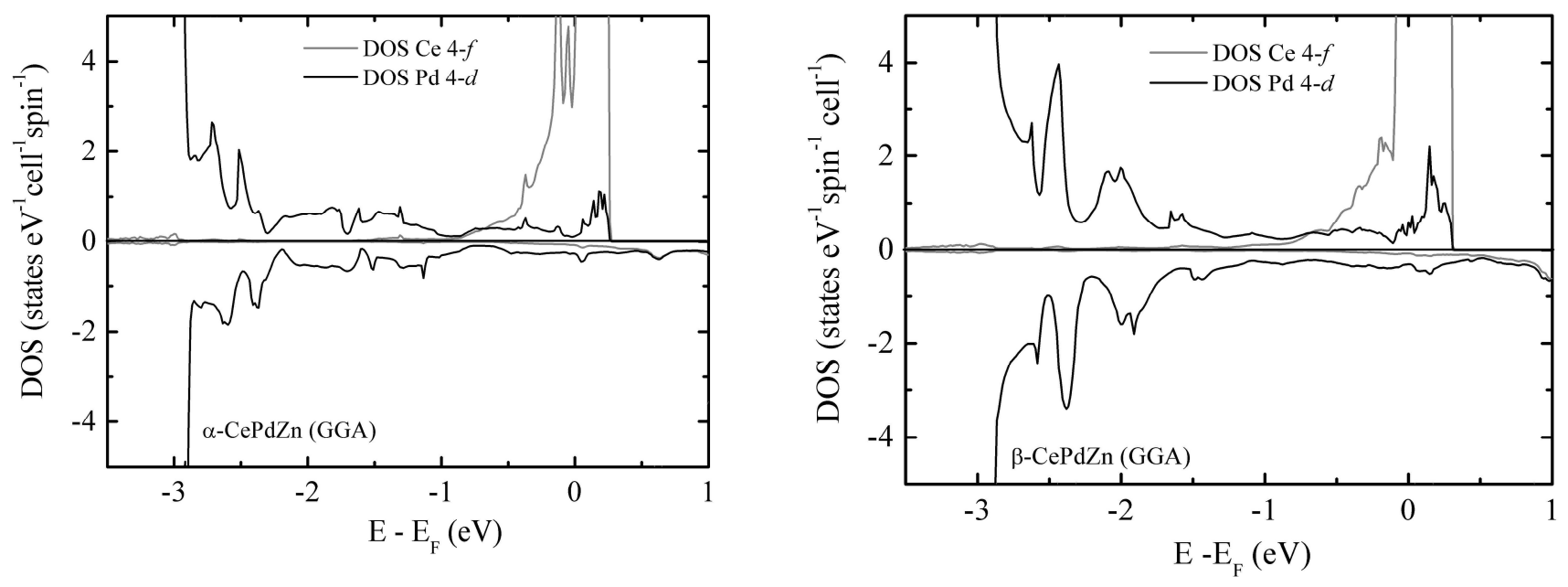

Fig. 6 Density of States for $\alpha-C e P d Z n$ and $\beta-C e P d Z n$ (GGA). 